\title{
Influence of microstructure and internal stress on the mechanical behavior of electroplated gold freestanding thin films
}

\author{
J.Martegoutte $^{13, a}$, C.Seguineau ${ }^{2}$, T.Fourcade ${ }^{24}$, C.Malhaire ${ }^{3}$, P.Martins ${ }^{3}$, J.-M.Desmarres ${ }^{1}$, and \\ X.Lafontan ${ }^{5}$ \\ 1 CNES, 18 av edouard Belin Bpi1414 31401 Toulouse Cedex 9, France \\ 2 NOVAMEMS, c/o CNES, DCT/AQ/LE, bpi 1414, 18 Av. Edouard Belin, 31401 Toulouse, France \\ 3 Université de Lyon, INSA-Lyon, INL, CNRS UMR 5270, Villeurbanne, F-69621, France \\ 4 LGP-ENIT, 47 Av. d'Azereix, BP 1629, 65016 Tarbes CEDEX, France \\ 5 INTESENS, 10 Avenue de l'Europe 31520 Ramonville, France
}

\begin{abstract}
Mechanical properties of freestanding electroplated gold thin films were studied in relationship to their geometrical and microstructural properties. Three different techniques of characterization were used: nanoindentation, bulge tests and microtensile tests. Results were compared to literature and also discussed according to physical phenomena related to the elaboration process of the specimens like seed layer exodiffusion or internal stress state. The observed plasticity and failure mechanisms were found to be in good agreement with the literature and are consistent with the microstructure. The measured Young's modulus is slightly higher than expected, and SIMS analysis is exploited to explain such a high value.
\end{abstract}

\section{Introduction}

The mechanical studies related to the microstrucural analysis and the development of new experimental methods are necessary to improve the reliability and the durability of Micro-ElectroMechanical Systems (MEMS). Evaluating the mechanical properties of thin film materials is an appropriate approach to understand failure mechanisms and to optimize the design of the current devices. Experimental approaches for the characterization of thin coatings deposited on substrate are mainly based on nanoindentation [1] and wafer curvature methods [2], whereas the characterization of freestanding coatings (expensively used in MEMS) remains more challenging. While bulge-test and pointdeflection methods are widely developed, microtensile experiment is currently less exploited despite quite promising results. Microstructural features, and physicochemical properties like the stress state or crystallography, have a direct consequence on mechanical properties. Those properties directly depend on the conditions of the elaboration process. Consequently, the direct link between the process conditions and the resulting mechanical properties could be established through intensive physicochemical analysis, including not only microstructural observations, but also internal stresses influence, impurities inclusions or crystallography changes for the highest energies. The present work focuses on two aspects: the use of nanoindentation, bulge-test and microtensile tests for the evaluation of the mechanical properties of a electroplated gold thin coatings either deposited on a Si substrate either as freestanding specimen, and the correlation of those properties with microstructure, internal stresses and porosity.

\footnotetext{
a e-mail: julien.martegoutte@cnes.fr
} 


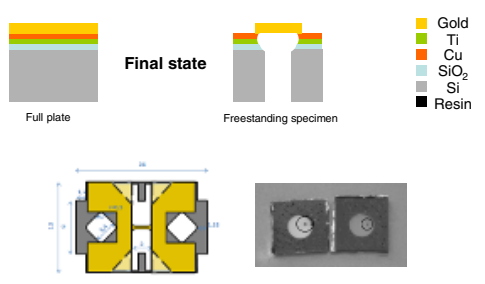

Fig. 1. On top : final state of specimens. On bottom: microtensile sample, and bulge-test membrane with burning defects rimmed in black

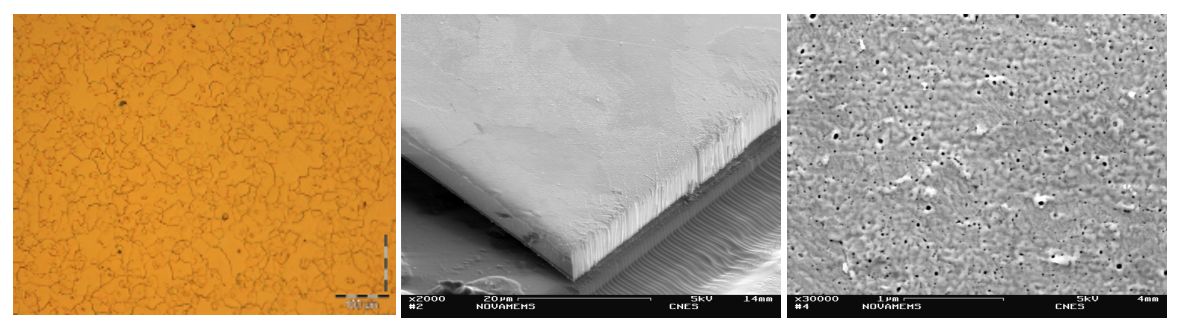

Fig. 2. Gold layer observations : Optical microscope surface picture - FIB cross section SEM picture - Surface SEM picture

\section{Electroplated gold : fabrication and characterization}

\subsection{Freestanding specimens: fabrication technologies}

Gold thin coatings were electrodeposited on a silicon wafer (double-sided polished, 4 inches, $400 \mu \mathrm{m}$ thick, P-type, $<100>$ orientation), and bulk micromachining was used afterward for releasing the specimens. Membranes and microtensile specimens were prepared on the same wafer. After a standard cleaning procedure based on $\mathrm{H}_{2} \mathrm{SO}_{4}: \mathrm{H}_{2} \mathrm{O}_{2}(3: 1)$, the wafer was thermally oxidized to form a $200 \mathrm{~nm}$ thick $\mathrm{SiO}_{2}$ layer on both sides. On the front side, a $50 \mathrm{~nm} / 50 \mathrm{~nm} \mathrm{Cu} / \mathrm{Ti}$ seed layer was deposited by thermal evaporation. A thick gold film $(5.4 \mu \mathrm{m})$ was then electrodeposited through a patterned SU-8 mold $(10 \mu \mathrm{m})$. After the SU-8 film removal, the backside thermal oxide was patterned before silicon etching. Freestanding specimens were released by etching the Si substrate from the backside by a Deep Reactive Ion Etching (DRIE) process. Finally, the remaining $\mathrm{SiO}_{2}, \mathrm{Ti}$ and $\mathrm{Cu}$ layers were chemically removed. to form a Au freestanding layer (fig. 1). After dicing, each $5 \mathrm{~mm}$ x $5 \mathrm{~mm}$ chip is dedicated to bulge test and contains an individual membrane with a diameter ranging from 2 to $3 \mathrm{~mm}$. The tensile specimens are $3 \mathrm{~mm}$ long (jauge length of $2 \mathrm{~mm}$ ), with a width varying from $200 \mu \mathrm{m}$ to $500 \mu \mathrm{m}$ (see figure 1), and are framed with silicon arms which were cut prior to the tensile test.

\subsection{Geometrical and physicochemical characterization}

Geometrical parameters were measured by a FOGALE optical interferometer. Thickness was mainly measured on wafer's edges and on tensile specimens grips. Width, length and diameter were directly measured on samples, and are summarized in table 2 in part 4.1.

The figure 2 represents optical pictures of the surface, a FIB cross section and a SEM picture of the surface. The observations reveal a pronounced columnar structure of the gold layer (diameters ranging from $10 \mu \mathrm{m}$ to $30 \mu \mathrm{m}$ ), with one grain through the thickness. Very small pores were observed with SEM-FEG. However, an image analysis shows that the porosity does not exceed $2 \%$, suggesting a limited impact on mechanical properties [3]. Concerning physicochemical properties, EDX analysis on FIB cross sections and SIMS analysis, performed on a circular membrane, have shown diffusion of atoms from the removed $\mathrm{Cu}$ seed layer through the film thickness. As for crystallographic characterization, the $<100>$ texture on columnar grains is widely present. However, a $18 \%<111>$ texture has been observed by Electron Back-Scattered Diffraction (EBSD). 


\section{Mechanical properties}

Three direct mechanical experiments have been used for a coupled evaluation of the mechanical properties: nanoindentation experiments on a gold layer deposited on the substrate, bulge-test and microtensile test which were performed on freestanding coatings, as described in part 2.

\subsection{Nanoindentation, Bulge-test and microtensile test : experimental set-up}

Nanoindentation experiments are performed on an Agilent XP nanoindenter (DCM head). The diamond tip is driven into the gold coating up to $6 \%$ of its thickness, in order to avoid substrate influence on the measures. A CSM device is used for a continuous evaluation of the reduced modulus, between 5.6 and 5.8\% of the coating thickness, by following the Oliver and Pharr model [1].

While nanoindentation is performed on gold coatings deposited on the substrate, bulge and tensile tests are realized on suitable freestanding specimens. Microtensile experiments are performed on a nomad experimental device already described elsewhere in [4] and [5]. Displacement speeds are set from $0.1 \mu \mathrm{m} \mathrm{s}^{-1}$ to $0.6 \mu \mathrm{m} \mathrm{s}^{-1}$, and the applied stress remains uniaxial, allowing direct evaluation of the mechanical properties.

Bulge test were performed on three circular membranes (the mean diameter is $2.5 \pm 0.4 \mathrm{~mm}$ and the global thickness is $5.4 \pm 0.1 \mu \mathrm{m}$. The applied pressure ranges from 0 to $500 \mathrm{mbar}$ in order to remain in the elastic domain, and the resulting membrane deflection is measured by using a WYCO NT1100 white-light interferometer microscope [6].

\subsection{Results}

Insofar as nanoindentation is based on a tip driven into the coating, the stress field is much more complex than in the case of bending or tensile experiments. As a result, equations ruling nanoindentation experiments involve a reduced modulus, which is composed from the elastic parameters of the diamond tip and of a biaxial modulus of the coating related to its Young's modulus by the formula : $E_{b}=E /\left(1-v^{2}\right)$. We obtained $E_{b}=83.7 \pm 2.7 \mathrm{GPa}$, and by assuming a Poisson's ratio value of 0.44 , the extracted Young's modulus from nanoindentation is then: $E=68.8 \pm 2.2 \mathrm{GPa}$. The nanoindentation hardness $\mathrm{H}$ is used to obtain an approximation of the yield stress $\sigma_{y}$ with the Tabor's relation: $H=3 \sigma_{y}$, which leads to $\sigma_{y}=312 \pm 6 \mathrm{MPa}$.

Concerning bulge-test, the mechanical properties are extracted from the relationship between the applied pressure $P$ and the corresponding maximum out-of-plane deflection $h$ of the bulged circular membrane [7]:

$$
P=\frac{2 E t}{(1-v) a^{4}} h^{3}+\frac{4 \sigma_{0} t}{a^{2}} h
$$

with $t$ the membrane thickness, $a$ the radius of the membrane, $E$ the Young's modulus, $v$ the Poisson's ratio and $\sigma_{0}$ the residual stress. Results show a perfect correlation between measured points and the analytical model. The intrinsic stress and the elastic modulus which have been extracted by following eq. 1 are presented in table 1 . The electroplated gold films revealed high tensile stresses with small uncertainties, and small elastic modulus values with important uncertainties. These characteristics are discussed in part 4.

Stress-strain curves related to microtensile test experiments are presented in figure 3 . Two distinct stages are observed on those curves. The linear part corresponds to the elastic response of gold samples. The slope value is the Young's modulus, which can be extracted with a linear regression. The second part of the curve describes the plastic behavior of the material. Several models can be used to characterize the elasticplastic behavior of this gold coating. A Ramberg - Osgood law (eq. 2) was chosen because of the very small strain involved (with a ultimate strain lower than 1\%). The Ramberg - Osgood's plastic parameters $\alpha$ and $\sigma_{R}$ are then more suitable to characterize the onset of plasticity than the conventional $\sigma_{y}=\sigma\left(\varepsilon_{p}=0.002\right)$.

$$
\varepsilon=\frac{\sigma}{E}+\alpha \frac{\sigma_{R}}{E}\left(\frac{\sigma}{\sigma_{R}}\right)^{N}
$$




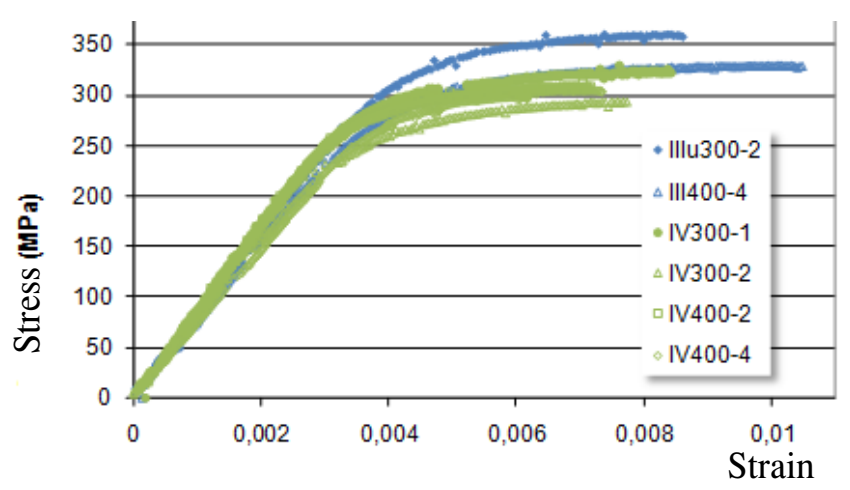

Fig. 3. $\sigma$ the axial stress as a function of $\varepsilon$ the strain

\begin{tabular}{|c|c|c|c|c|c|}
\hline Experiment & $\mathbf{E}(\mathrm{Gpa})$ & $\boldsymbol{\sigma 0}$ (Mpa) & $\boldsymbol{\sigma y}(\mathrm{Mpa})$ & $\mathbf{N}$ & $\boldsymbol{\varepsilon}_{\mathbf{R}}$ \\
\hline Nanoindentation & $68,8 \pm 2,2$ & & $312 \pm 6$ & & \\
Bulge-test & $62 \pm 29$ & $184 \pm 17$ & $310 \pm 25$ & $17,7 \pm 2,1$ & $0,1 \%$ \\
Micro-tensile & $80.1 \pm 4,8$ & & & \\
\hline
\end{tabular}

Table 1. Extracted mechanical parameters from different cross test

where we've chosen $\alpha=\frac{1}{2}$ that is to say that $\sigma_{R}=\sigma\left(\frac{\varepsilon_{p}}{\varepsilon_{e}}=\frac{1}{2}\right)$. It appears that in this case, $\sigma_{R}(\alpha=$ $\frac{1}{2} \approx \sigma_{Y}(\varepsilon=0.002$ Young's modulus, representative plastic stress and work-hardening coefficient are shown in table 1 . Those parameters have been calculated by logarithmic regression on the equation 2.

\section{Discussion}

\subsection{Literature material comparison}

Four distinct gold materials, studied by as many different authors are used for providing comparisons: Baek et al. [8], Espinosa et al. [9], Lee et al. [10] and Kim et al. [11]. The table 2 summarizes these materials characteristics. The deposit technologies are not the same from one author to another (sputtering, arc evaporation and electrodeposition), and the resulting materials characteristic are quite different. Our purpose is here to identify relevant material parameters for establishing a correlation with mechanical properties. The magnitude order of grain size, for example, varies widely from $10 \mathrm{~nm}$ to $10^{5} \mathrm{~nm}(20 \mu \mathrm{m}$ in our case): a Hall-Petch effect on the yield strength could be expected [12].

The geometrical parameters describing the specimens are also quite different, insofar as mechanical characterization is obtained through a wide range of set-ups. Thickness remains nonetheless one of the most critical. Indeed, that characteristic coating length is of the same order of magnitude of the microstructural features dimensions, resulting in a possibly strong interaction with the mechanical properties, known as "size effect" [13].

Such an effect is expected because the electroplated gold samples have a very large grain size, with a columnar microstructure : there is only one grain in the thickness. Geometrical and microstructural parameters are compared to bibliography in part 4.4 for this purpose.

\subsection{Mechanical properties measurements: uncertainties discussion}

Evaluating the uncertainties associated with the different methods of characterization requires a precise knowledge of the geometry of the test structures (especially for self-standing specimens) and of the equations ruling the analytical models. As a consequence, they can greatly vary from one kind of experiment to another. 


\begin{tabular}{|c|c|c|c|c|c|c|c|}
\hline Bibliography & Width $(\mu \mathrm{m})$ & Grain number & Grain size $(\mathrm{nm})$ & Thickness $(\mu \mathrm{m})$ & Grain number & Grain size $(\mathrm{nm})$ & Texturation \\
\hline \multirow{4}{*}{ Espinosa 2004} & 2,5 & \begin{tabular}{ll|}
9 & 1
\end{tabular} & $281 \pm 31$ & \multirow{4}{*}{$\begin{array}{c}0,3 \\
0,5 \\
1\end{array}$} & \multirow{4}{*}{$\begin{array}{rl}1.5 & 0.5 \\
2.25 & 0.75 \\
4.5 & 1.5\end{array}$} & $200 \quad 67$ & \multirow{4}{*}{$<111>$} \\
\hline & 5 & 18 & $281 \pm 31$ & & & 22274 & \\
\hline & 10 & 36 & $281 \pm 31$ & & & $222 \quad 74$ & \\
\hline & 20 & 73 & $276 \pm 26$ & & & & \\
\hline Lee 2006 & $\begin{array}{l}100 \\
300\end{array}$ & $\begin{array}{cl}3.5 & 1.5 \\
11 & 4 \\
\end{array}$ & $30 \pm 10$ & $99.8 \pm 7.1 \mathrm{~nm}$ & $3.75 \quad 1.25$ & 30 & $\begin{array}{l}<100>\text { préférentielle } \\
\text { on orignial material }\end{array}$ \\
\hline Baek 2005 & 100 & $133 \pm 44$ & $750 \pm 250$ & $\begin{array}{l}3.35 \pm 0.05 \\
4.08 \pm 0.11\end{array}$ & $\begin{array}{ll}4.5 & 1.5 \\
5.4 & 1.8\end{array}$ & $750 \pm 250$ & Unknown \\
\hline Kim 2006 & $24 \mu \mathrm{m}$ (diameter) & $\begin{array}{c}120 \pm 12 \\
80 \pm 4 \\
68.5 \pm 7 \\
60 \pm 6 \\
56 \pm 5 \\
60 \pm 6\end{array}$ & $\begin{array}{l}200 \pm 20 \\
300 \pm 15 \\
350 \pm 35 \\
400 \pm 40 \\
430 \pm 43 \\
400 \pm 40\end{array}$ & $24 \mu \mathrm{m}$ (diameter) & $\begin{array}{c}120 \pm 12 \\
80 \pm 4 \\
68.5 \pm 7 \\
60 \pm 6 \\
56 \pm 5 \\
60 \pm 6\end{array}$ & $\begin{array}{l}200 \pm 20 \\
300 \pm 15 \\
350 \pm 35 \\
400 \pm 40 \\
430 \pm 43 \\
400 \pm 40\end{array}$ & Originally $<111>$ \\
\hline & & $\begin{array}{c}80 \pm 24 \\
44 \pm 4 \\
28 \pm 4 \\
22 \pm 4 \\
14 \pm 2 \\
\end{array}$ & $\begin{array}{c}300 \pm 90 \\
540 \pm 54 \\
850 \pm 127 \\
1070 \pm 214 \\
1650 \pm 247\end{array}$ & & $\begin{array}{c}80 \pm 24 \\
44 \pm 4 \\
28 \pm 4 \\
22 \pm 4 \\
14 \pm 2 \\
\end{array}$ & $\begin{array}{c}300 \pm 90 \\
540 \pm 54 \\
850 \pm 127 \\
1070 \pm 214 \\
1650 \pm 247\end{array}$ & $<100>$ after annealing \\
\hline Present work & $\begin{array}{l}293 \pm 3 \\
399 \pm 1\end{array}$ & $\begin{array}{cc}15 & 7 \\
20 & 10 \\
\end{array}$ & 2000010000 & $\begin{array}{ll}5.4 & 0.2 \text { (bulge test) } \\
& 4.10 .2\end{array}$ & 1 & $\begin{array}{ll}5.4 & 0.2 \\
4.1 & 0.2 \\
\end{array}$ & $<100>$ and $18 \%<111>$ \\
\hline
\end{tabular}

Table 2. Geometrical and microstructural parameters of gold thin films from four different sources. In grey: calculated parameters from explicit dataset

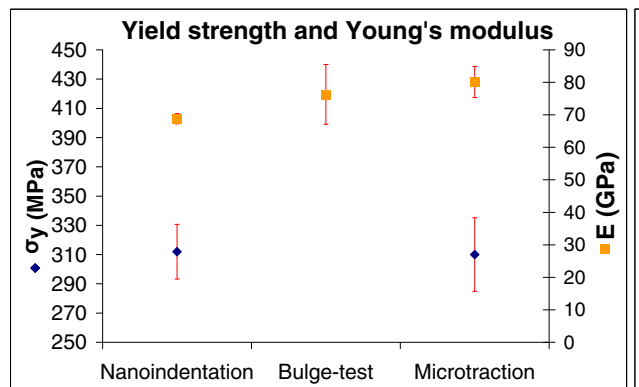

(a) : Mechanical properties from cross tests

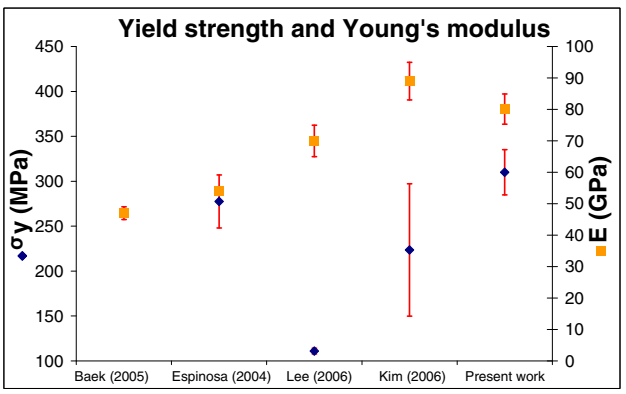

(b) : Mechanical properties from literature

Fig. 4. Young's modulus and yield strength of electroplated gold from cross tests, and from different gold freestanding literature samples. The present work item corresponds to microtensile experiments results

Concerning the Young's modulus, nanoindentation seems to give the smallest uncertainty (fig. 4(a)). But that result doesn't take into account the indetermination of the Poisson's ratio: an average uncertainty of 5\% will result in a deviation on the Young's modulus of more than $13 \%$. Besides, other authors have used methods based on freestanding specimen for the mechanical characterization. The nanoindentation results should be cautioulsly exploited for the following comparisons.

As for the two kind of techniques involving freestanding specimens, the bulge-test leads to the more important uncertainty on elastic properties. From a modeling point of view, the diameter of the membrane is at the power four in the first term, which is used for the determination of $E$ (eq. 1). Experimental considerations could also explain such a wide deviation: only low pressures were applied on the membranes because of adhesion defects of our gold coatings. The resulting $P(h)$ curve is quite linear, where the Young's modulus is deduced from the non-linear part. Nevertheless, bulge-test with small out of plane deflections is well suited for the residual stress determination, which is based on the linear part of the curve.

If considering tensile experiments and the equation 2, dimensional parameters are linearly involved. That is to say that tensile loadings are less sensitive towards uncertainties on geometrical parameters than bending experiments. Besides, Young's modulus determination is obtained from a linear relation between stress and strain. The results of that particular experiments have been therefore chosen for a relevant comparison with literature, the calculated $\sigma_{y}$ value being the same as the $\sigma_{R}$ value. Yet, even if tensile experiments are not influenced by tensile internal stresses thanks to a specific experimental protocol, it is not adapted to provide reliable measurements of internal stresses, contrary to the bulge test. 


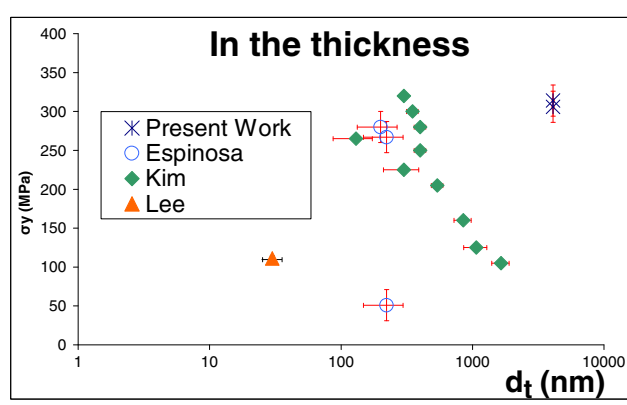

(a) : Yield stress as a function of grain size in the thickness

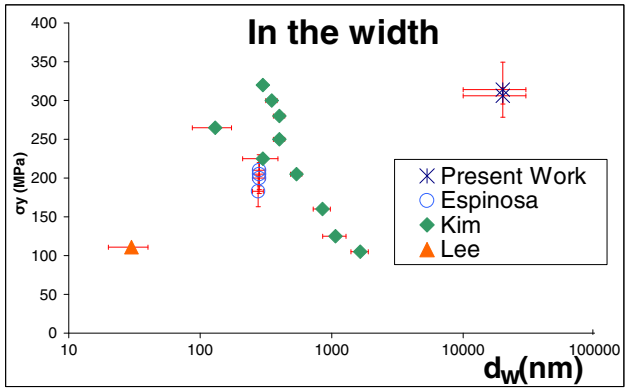

(b) : Yield stress as a function of grain size in the width

Fig. 5. Yield strength as a function of grain size, determined in the direction in the thickness $\left(d_{T}\right)$ and in the direction of the width $\left(d_{W}\right)$

\subsection{Literature comparison}

The results of this study are compared with those published by the four authors listed before. All of them have performed microstructural observations. Young's modulus and yield strength extracted from the literature and results of the present work, provided by microtensile experiments, have been compared in figure 4(b). First, noticeable differences in Young's modulus evaluation are highlighted, although differences between uncertainties make that analysis difficult. Espinosa et al. and Baek et $a l$., which have not performed annealing on their samples, do not have very important uncertainties, instead of Kim who has changed microstructure and texture by annealing his samples. The yield stress is also greatly impacted by these thermal effects.

Variations in grain size can be used to highlight the Hall-Petch effect on the yield stress. A comparison is realized on the yield stress, as plotted in fig. 5. Concerning Espinosa et al. values, they have chosen to characterize the plasticity occurrence with the first onset of non linearity. The values plotted on fig 5 are estimated from graphical interpretation made on [9]. In both figures 5(a) and (b), a HallPetch effect could be observed: the yield stress increases with increasing grain size until a possible critic grain size about $200 \mathrm{~nm}$. For smaller grains, Lee deduced a smaller $\sigma_{y}$ from FEM performed on nanoindentation experiments, which could be related to the "Hall-Petch inverse" [14]. However, in our case, evaluated values do not fit with the Hall-Petch trends. The figure 6 represents the yield stress as a function of the adimensional grain number through the thickness and through the width. On the fig 6(a), two trends appear: a clear decrease of yield stresses from 1 grain to nearly 5 grains and then an increase to nearly 80 grains in the thickness. In this case, our results are in good concordance with these two trends, particularly with Espinosa who shows that the less the number of grains in the thickness is, the higher the yield stress is measured. On the contrary, the yield stress does not seem correlated with the adimensional grain diameter in width $\mathrm{W}$ in fig 6(b), even if our data are comparable with Espinosa's. The comparison between fig 6(a) et fig. 6(b) shows as expected that $\sigma_{y}$ seems to be clearly determined by thickness.

To conclude, the grain size parameter is pertinent to see Hall-Petch effects on samples with identical dimensions. However, comparing samples with different dimensions requires to divide by the thickness or width to understand geometrical size effects. The resulting adimensional parameter grain size $\mathrm{T}$ appears to be necessary to compare size effects in samples with different geometries, particularly for the design optimization of these coatings.

\subsection{Microstructure - mechanical properties correlation}

Relevant geometrical criteria have been studied, and trends have also been established between them in order to see their influence on both samples and microstructure. To complete the material study, the physicochemical properties of the material are taken into account, including the chemical composition, microstructure and crystallographic organization of the material. 


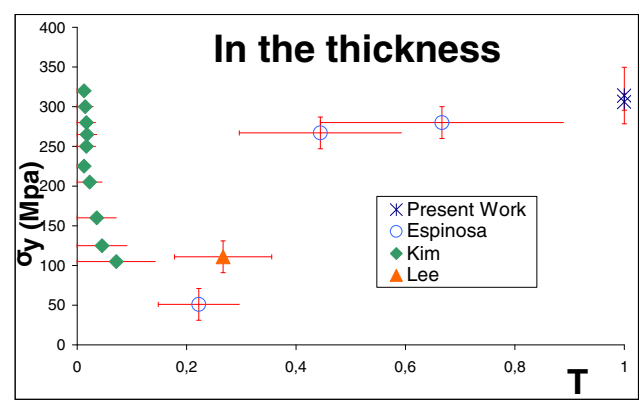

(a) : Yield stress as a function of T

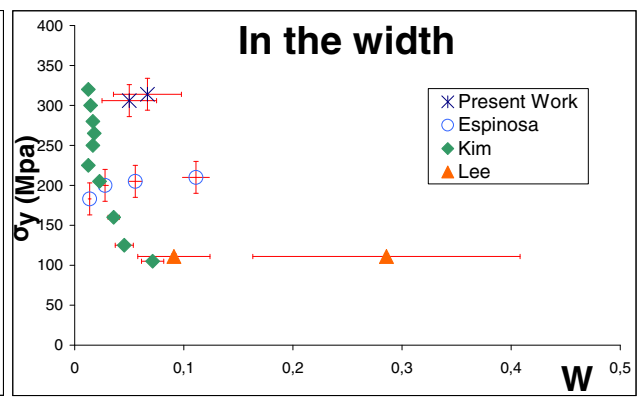

(b) : Yield stress as a function of W

Fig. 6. Yield strength as a function of adimensional grain size in thickness $\left(T=\frac{d_{T}}{t}\right)$, and in the width $\left(W=\frac{d_{W}}{w}\right)$, with $t$ the thickness and $w$ the width

As seen on figure 4(b), the Young's modulus of the present work ( $E=80.1 \mathrm{GPa})$ is quite higher than Espinosa's, Lee's and Baek's. That can be explained in several ways. First, the modulus value is close to bulk counterpart, which seems in good agreement with the higher thickness of the layer. However, its columnar grain structure with one grain in the thickness suggests a thin coating behavior. Thus, a $<111>$ texture was observed by EBSD experiments and quantified, by image analysis, at $V_{<111>} \approx$ 0.18. That observation has also been noticed by Kim et al., who explains his high modulus with this texture effect. Assuming $E_{<100>}=43 \mathrm{GPa}$ and $E_{<111>}=117 \mathrm{GPa}$ [15], and assuming $V_{<100>}+V_{<111>}=$ 1, a Reuss and Voigt mix law, described in formula 3 and 4, may explain that high modulus value:

$$
\begin{gathered}
E=E_{<111>} V_{<111>}+E_{<100>} V_{<100>} \text { for the Voigt limit } \\
E=\left(\frac{V_{<111>}}{E_{<111>}}+\frac{V_{<100>}}{E_{<100>}}\right) \text { for the Reuss limit }
\end{gathered}
$$

Numerical applications give the Voigt and Reuss limits : 49GPa $\leq E \leq 56 G P a$, which are largely lower than measured values. Consequently, the presence of $<111>$ texture is not sufficient to explain our high elastic modulus. Another explanation is the presence of solid solution of copper $(\mathrm{Cu})$ that has been pointed out by a SIMS analysis into the bottom of the gold layer (diffusion from the seed layer). As the copper Young's modulus is reported to be nearly $120 \mathrm{GPa}$, it could explain the higher measured modulus of gold samples. However, because of the copper capability to mix and form orthorhombic phase, X-ray Diffraction (XRD) experiments should be performed to know the lattice parameters. In parallel, copper concentration through the thickness should be measured to identify crystallographic phases. Finally, if it is possible that both gold surface texture and $\mathrm{Cu}$ diffusion have a significant influence on the Young's modulus value, the distinction between them remains difficult.

The comparison of the plastic properties provides an insight about the impact of process variations on the mechanical behavior of thin coatings, more particularly for reliability statements. The intrinsic residual stress should be also taken into account insofar as it could modify the measured yield stress. Thus, because freestanding structures could partially release elastic strain, some differences can occur depending on the setup used for mechanical characterization. In our case, the bulge-test is the best way to estimate the residual stress: $\sigma_{0}=200 \mathrm{MPa}$. The yield stress, measured by tensile experiments, is the highest of the reported values (fig. 4(b)). However, Lee's yield stress value comes from nanoindentation extrapolation, unlike the other authors, and involves different stress states under the tip. As for Kim results, the comparison is limited insofar as internal stresses and strain hardening are limited by annealing.

Finally, concerning failure mechanisms, electroplated gold films have shown a classic ductile failure (as all fcc metals) because failure microvoids were initiated on nano-pores. The failure occurs after a shallow necking. Indeed, the large grain size and their columnar shape did not allow sliding of grains; as soon as a grain boundary is weakened, the crack initiates at that boundary. Ultimate strain $\left(\varepsilon_{r} \approx 0.8 \%\right)$ agrees with literature [9], and particularly Espinosa et al. whom ultimate strains do not 
exceed $\varepsilon_{r}=1 \%$. Nonetheless, the random localization of the crack on the tensile specimen proves that such an early failure could not be imputed to our experimental setup. Despite the small plasticity, shear bands have been observed, with an angle of $60^{\circ}$ toward the stress direction. This is partially in agreement with results of Espinosa et al. who observed $90^{\circ}$ and $60^{\circ}$ shear bands. Texture may explain the shift in our shear bands compared with the maximal shear stress $45^{\circ}$ direction. This phenomena has been studied by Shih et al. [16]: in rolled fcc metals, a theoretical explanation, by calculating Taylor's factors, was made to give the influence of $\{110\}<001>,\{110\}<112>$ and $\{112\}<111>$ texture on their observed $35^{\circ}$ shear bands.

\section{Conclusion}

Mechanical properties of electroplated freestanding structures have been measured by three different approaches. Bulge test is currently suitable for the characterization of elastic properties, including tensile internal stress, whereas tensile experiments allow us to reach large deformation and then are currently more adapted to evaluate plastic properties like yield stress or strain hardening. Quite high, if compared with literature, elastic modulus may be due to the combination of the $18 \%<111>$ texture and of the diffusion of $\mathrm{Cu}$ atoms. As for yield stress, its high value could be explained through analysis reported elsewhere. Grain size parameter alone fails to explain that high value. It must be used coupled with geometrical parameters to fully take into account size effects. That is why we propose the use of the adimensional thickness grain size (T) in order to compare the mechanical behavior of coatings with varying thickness. The use of three different approaches for the evaluation of the mechanical properties shows a good agreement between them. Moreover, the lack of correlation between $\sigma_{y}$ and either $d_{W}$ or $W$ proves the relevance of the experimental approaches to evaluate the mechanical properties from specific freestanding metallic thin layer specimens. Indeed, the no bias seems to be inserted by design of specimens as long as thickness is similar to the materials one to be characterized. Failure mechanism are also similar to literature's counterpart. The reduced number of grains in the thickness implies a limited plastic strain before failure, without relevant necking. Complementary works are currently under progress to apply the Shih's approach in order to explain the $60^{\circ}$ shear bands.

\section{References}

1. W.C. Oliver, G.M. Pharr, Journal of Material Research 19, (2004) 3-20

2. G.C.A.M. Janssen, Thin Solid Films 515, (2007) 6654-6664

3. A.R. Boccaccini, Z. Fan, Ceramics International 23, (1997) 239-245

4. M. Ignat, L. Debove, C. Josserond, Bulletin SMFE 14, (1996) 10-13

5. C. Malhaire, C. Seguineau, M. Ignat, C. Josserond, L. Debove, S. Brida, J.M. Desmarres, X. Lafontan, Review of Scientific Instruments 80, (2009) 10-13

6. P. Martins, C. Malhaire, S. Brida and D. Barbier, Microsyst Technol 15, (2009) 1343-1348

7. D. Xu, K. M. Liechti, Experimental Mechanics 50, (2010) 217-225

8. C.W. Baek, Y.K. Kim, Y. Ahn, Y.H. Kim, Sensors \& Actuators: A. Physical 117, (2005) 17-27

9. H.D Espinosa, B.C Prorok, B. Peng, Journal of the Mechanics and Physics of Solids 52, (2004) 667-689

10. D. Lee, X. Wei, X. Chen, M. Zhao, S.C. Jun, J. Hone, E.G.Herbert, W.C. Oliver, J.W. Kysar, Scripta materialia 56, (2006) 437-440

11. K.S. Kim,J.Y. Song, E.K. Chung, J.K Park, S.H Hong, Mechanics of Materials 38, (2006) 119-127

12. A.H. Chokshi, A. Rosen, J. Karch, H. Gleiter, Scripta Metallurgica 23, (1989) 1679-1683

13. E. Arzt, Acta materialia 46, (1998) 5611-56261

14. M.A. Meyers, A. Mishra, D.J. Benson, Progress in Materials Science 51, (2006) 427-556

15. T.H. Courtney, McGraw-Hill New York (1990) 179, 182, 183, 184, 210

16. C. Shih-Chin, H. Duen-Huei, C. Yun-Kie, Acta Metallurgica 37, (1989) 2031-2033 